Résumés des conférences et travaux

\title{
Le débat entre nominalistes et réalistes à la fin du Moyen Âge
}

Alessandro D. Conti

\section{(2) OpenEdition}

\section{Journals}

Édition électronique

URL : https://journals.openedition.org/ashp/3831

DOI : 10.4000/ashp.3831

ISSN : 1969-6310

Éditeur

Publications de l'École Pratique des Hautes Études

Édition imprimée

Date de publication : 1 septembre 2020

Pagination : 238-241

ISSN : 0766-0677

\section{Référence électronique}

Alessandro D. Conti, « Le débat entre nominalistes et réalistes à la fin du Moyen Âge », Annuaire de

I'École pratique des hautes études (EPHE), Section des sciences historiques et philologiques [En ligne],

151 | 2020, mis en ligne le 09 juillet 2020, consulté le 21 septembre 2021. URL : http://

journals.openedition.org/ashp/3831; DOI : https://doi.org/10.4000/ashp.3831 


\title{
LE DÉBAT ENTRE NOMINALISTES ET RÉALISTES À LA FIN DU MOYEN ÂGE
}

\author{
Conférences de M. Alessandro D. ConTI, \\ professeur à l'université de L'Aquila (Italie), \\ directeur d'études invité
}

Le réalisme et le nominalisme ont constitué la principale alternative philosophique élaborée entre la fin du Moyen Âge et le début de l'époque moderne au sujet de la structure et de l'ordre de la réalité ainsi que de la nature et de la fonction de l'esprit et du langage. De manière très simplifiée, l'on peut dire que les réalistes admettaient (d'une certaine façon) l'existence extra-mentale de natures (ou formes ou essences) communes, alors que les nominalistes la niaient. Les réalistes pensaient que la table des catégories décrite par Aristote dans le traité du même nom répartissait des entités réelles sur la base de critères ontologiques; les nominalistes, quant à eux, soutenaient que les dix catégories aristotéliciennes répartissaient les termes (mentaux, vocaux et écrits) de notre langage selon des critères sémantiques, et que dans la réalité il n'existait que deux (ou trois) types différents d'entités : les substances, les qualités et (peut-être) les quantités. Les réalistes croyaient que le monde, la pensée et le langage étaient structurellement homologues, et qu'ils se reflétaient l'un l'autre. Les nominalistes distinguaient nettement entre les choses existant dans le monde extra-mental et leurs relations réciproques, d'une part, et les différentes manières et formes dont notre esprit nous permet de les penser et d'en parler, de l'autre; de sorte que, pour eux, le monde, la pensée et le langage étaient des systèmes logiquement indépendants. Le conflit entre ces deux paradigmes opposés a certainement été le ressort le plus important dans l'évolution de la logique, de la métaphysique et de la gnoséologie entre la fin du Moyen Âge et le début de l'époque moderne, et il a produit un affinement progressif des modalités d'argumentation et des procédures démonstratives effectivement employées dans la défense de positions de plus en plus complexes et nuancées.

\section{La polémique entre Ockham et Burley au sujet de la supposition, des universaux et des catégories}

Guillaume d'Ockham et Walter Burley (1275 - après 1344) sont probablement les penseurs les plus remarquables du XIV ${ }^{\mathrm{e}}$ siècle parmi les champions de l'approche philosophique respectivement nominaliste et réaliste. Leur activité polémique se situe dans la première moitié du XIV ${ }^{\mathrm{e}}$ siècle - une période décisive, pendant laquelle la diffusion des théories d'Ockham provoqua un conflit acharné entre les partisans de la vieille thèse réaliste, comme Burley, et les défenseurs de la nouvelle voie nominaliste, comme Ockham lui-même. Burley usa de toutes ses ressources intellectuelles et 
de son autorité pour combattre la nouvelle tendance nominaliste, et il fut le premier penseur qui essaya d'éliminer par une stratégie adéquate les apories, soulignées par Ockham, de la théorie traditionnelle des catégories et des universaux. Après 1324, dans ses écrits Burley soutint que (1) les universaux, compris comme des formes générales, existent pleinement en dehors de l'esprit et sont réellement distincts des individus dans lesquels ils sont présents et dont ils sont prédiqués; (2) le monde extérieur contient des propositions réelles qui sont les significata des phrases vraies; (3) il y a une distinction réelle entre les dix catégories, dont chacune était considérée par Burley comme une chose dans le sens propre du mot. Dans cette conférence M. Alessandro Conti a présenté les caractéristiques principales des ontologies opposées de Burley et d'Ockham, et il s'est efforcé de montrer les prémisses sémantiques de leurs doctrines et de décrire le développement de leur débat sur les catégories et les universaux. Tout d'abord, il a esquissé les aspects essentiels de la doctrine réaliste de Burley sur les catégories et les universaux telle qu'il l'élabora au début du XIV ${ }^{\mathrm{e}}$ siècle. Ensuite, il a résumé l'attaque portée par Ockham à l'encontre de cette théorie traditionnelle et il a exposé brièvement ses idées sur les catégories et les universaux. Troisièmement, il a considéré la réponse de Burley et il a illustré les traits fondamentaux de sa dernière version du réalisme. Enfin, il a comparé leurs systèmes afin de montrer les points communs et les différences entre eux.

\section{Les critiques de Wyclif à l'encontre d'Ockham : logique, métaphysique et théologie}

Parmi les adversaires d'Ockham, l'un des plus intelligents et certainement le plus influent fut John Wyclif (1330 environ - 1384). Bien qu'il ait achevé son premier ouvrage académique (le traité De logica) plusieurs années après la mort d'Ockham, ses théories philosophiques fondamentales peuvent être lues, en un sens, comme une réponse à Ockham. En fait, pendant sa jeunesse Wyclif n'épousa pas la cause du réalisme; bien au contraire, au début de ses études il sympathisait avec les thèses nominalistes. On ne sait pas à quel moment il faut situer son revirement, mais ce fut sûrement avant 1360 (date probable de composition de son premier ouvrage), car dans tous ses écrits qui nous sont parvenus il souligne constamment et très nettement son adhésion au réalisme. L'on ne sait pas ce qui a provoqué ce revirement, mais il est clair qu'il pensait que la validité et l'universalité de notre connaissance ne peuvent s'expliquer et se justifier que sur la base d'un isomorphisme étroit entre la pensée, le langage et le monde. Cette idée était opposée aux croyances des nominalistes, qui distinguaient les choses en tant qu'elles existent dans le monde extra-mental et les notions et les schémas par lesquels nous comprenons et désignons les choses. D'après Ockham, il n'y a dans le monde que des individus appartenant à deux genres différents, c'est-à-dire la substance et la qualité; les concepts par lesquels ils sont saisis et signifiés, en revanche, sont à la fois individuels et universels, et de dix genres différents (les dix catégories d'Aristote). Les relations par lesquelles nous relions nos concepts dans une proposition ne correspondent pas aux liens réels qui d'une certaine manière relient les éléments individuels dans un état de choses. Par conséquent, notre connaissance ne reproduit pas le monde et ses objets : elle les concerne seulement. 
M. A. Conti a d'abord présenté les thèses principales de Wyclif sur la logique, la métaphysique et la théologie, ainsi qu'un choix de thèmes où son profond désaccord avec Ockham est particulièrement manifeste. Pour ce qui est de la logique, M. A. Conti s'est occupé des notions d'identité, signification, supposition, prédication et vérité. Quant à la métaphysique, il s'est penché sur les problèmes des universaux et des catégories. Il a traité également de l'influence exercée par leurs différentes ontologies sur leurs systèmes théologiques, tout particulièrement en ce qui concerne les doctrines de l'exemplarisme divin et de la création. Afin d'illustrer le rapport polémique entre les théories de Wyclif et celles d'Ockham, M. A. Conti s'est concentré non seulement sur les différentes thèses qu'ils développèrent, mais également, dans la mesure du possible, sur les arguments élaborés par Wyclif en réponse aux positions d'Ockham.

\section{Johannes Scharpe et les réalistes d'Oxford}

La philosophie de Wyclif exerça une très grande influence sur les formes du réalisme tardo-médiéval. Ses intuitions sur les universaux, la prédication et les catégories jouèrent un rôle décisif dans la logique et la métaphysique de plusieurs philosophes, tout particulièrement de ceux que l'on appelle "réalistes d'Oxford »: les Anglais Robert Alyngton († 1398), William Milverley, William Penbygull († 1420), Roger Whelpdale ( $\dagger$ 1423), et John Tarteys, l'Allemand Johannes Scharpe ( $\dagger$ après 1415), et l'Italien Paul de Venise (1369-1429). Selon ces penseurs, les universaux et les individus étaient réellement identiques mais formellement distincts, et la prédication était une relation réelle entre les choses. Ils modifièrent partiellement la doctrine de Wyclif et introduisirent un nouveau type de prédication, qu'ils qualifièrent de « prédication par essence » (secundum essentiam), sur la base d'une identité partielle entre les entités indiquées par le sujet et le prédicat. Ils redéfinirent également les catégories traditionnelles post-aristotéliciennes de prédication essentielle et accidentelle dans les termes de cette identité partielle. Trois d'entre eux, Penbygull, Scharpe et Paul de Venise, à partir de la caractérisation de l'identité et de la distinction donnée par Wyclif, formulèrent de nouvelles définitions de ces relations transcendantales, lesquelles constituent les outils principaux qu'ils utilisèrent pour la construction de leurs systèmes métaphysiques.

\section{Les derniers grands protagonistes : Paul de Venise contre Buridan et Marsile d'Inghen}

À la lumière du tableau général que M. A. Conti a brossé dans les leçons précédentes, dans cette dernière conférence il a souhaité présenter quelques-unes des objections que Paolo Nicoletti de Venise, le logicien et philosophe italien le plus important du $\mathrm{XV}^{\mathrm{e}}$ siècle, soulève contre les nominalistes du Moyen Âge tardif, notamment contre Jean Buridan et Marsile d'Inghen, surtout à propos des universaux et des catégories. M. A. Conti a essayé de montrer en même temps comment Nicoletti élabore ses conceptions essentiellement pour contourner les points faibles, soulignés par Ockham dans ses écrits, de la version traditionnelle du réalisme modéré répandue entre le $\mathrm{XIII}^{\mathrm{e}}$ et le $\mathrm{XIV}^{\mathrm{e}}$ siècle. Au vu de l'ampleur de la production philosophique de 
Nicoletti, il n'a considéré que la sixième et dernière partie de la Summa philosophiae naturalis (1408), la Quaestio de universalibus (rédigée vraisemblablement pendant la deuxième décennie du $\mathrm{Xv}^{\mathrm{e}}$ siècle), la Lectura super libros Metaphysicorum (rédigée elle aussi vraisemblablement au début des années $20 \mathrm{du} \mathrm{XV}^{\mathrm{e}}$ siècle) et l'Expositio super Universalia Porphyrii et Artem Veterem Aristotelis (terminée en 1428, c'est-àdire un an avant sa mort, et contenant les commentaires par lemmes sur l'Isagoge de Porphyre, sur les Catégories d'Aristote et sur le Liber sex principiorum), qui se veut une véritable somme des doctrines médiévales sur les universaux et les catégories. 